\title{
PRESENTATION OF STATISTICAL CONCEPTS WITH DYNAMIC GRAPHICS AND SIMULATIONS IN R
}

\author{
Andrej Blejec \\ National Institute of Biology, Slovenia \\ andrej.blejec@nib.si
}

Understanding statistical concepts is important for proper use of statistics. The idea of using simulations and dynamic graphics to foster understanding of statistical concepts is not new. In recent years, $\mathrm{R}$ became the lingua franca for statistical data analysis. While $\mathrm{R}$ graphical devices are not meant for display of animated graphics, my aim is to use base R graphics for display of animated graphical sequences. To enable dynamic graphics in R, I developed a package animatoR, which supports smooth transitions of graphical elements and simplifies preparation of animated displays. I will show some animations that can be useful for statistics teaching and present basic features of the animato $R$ package. The animatoR package is freely available at https://github.com/ablejec/animatoR. 